\title{
Short communication: Suitability of fluorescence spectroscopy for characterization of commercial milk of different composition and origin
}

\author{
M. P. Ntakatsane, ${ }^{\star} \dagger$ X. Q. Yang, ${ }^{*}$ M. Lin, $\neq$ X. M. Liu, ${ }^{, 1}$ and P. Zhou ${ }^{\star 1}$ \\ *State Key Laboratory of Food Science and Technology, School of Food Science and Technology, Jiangnan University, Wuxi, \\ Jiangsu Province 214122, China \\ †Department of Animal Science, National University of Lesotho, Roma 180, Lesotho \\ ¥Division of Food Systems and Bioengineering, University of Missouri, Columbia 65211
}

\begin{abstract}
Thirteen milk brands comprising 76 pasteurized and UHT milk samples of various compositions (whole, reduced fat, skimmed, low lactose, and high protein) were obtained from local supermarkets, and milk samples manufactured in various countries were discriminated using front-face fluorescence spectroscopy (FFFS) coupled with chemometric tools. The emission spectra of Maillard reaction products and riboflavin $(\mathrm{MRP} / \mathrm{RF}$; 400 to $600 \mathrm{~nm}$ ) and tryptophan (300 to $400 \mathrm{~nm}$ ) were recorded using FFFS, and the excitation wavelengths were set at $360 \mathrm{~nm}$ for $\mathrm{MRP} / \mathrm{RF}$ and $290 \mathrm{~nm}$ for tryptophan. Principal component analysis (PCA) was applied to analyze the normalized spectra. The PCA of spectral information from $\mathrm{MRP} / \mathrm{RF}$ discriminated the milk samples originating in different countries, and PCA of spectral information from tryptophan discriminated the samples according to composition. The fluorescence spectral data were compared with liquid chromatography-mass spectrometry results for the glycation extent of the milk samples, and a positive association $\left(\mathrm{R}^{2}=0.84\right)$ was found between the degree of glycation of $\alpha$-lactalbumin and the $\mathrm{MRP} / \mathrm{RF}$ spectral data. This study demonstrates the ability and sensitivity of FFFS to rapidly discriminate and classify commercial milk with various compositions and processing conditions.
\end{abstract}

Key words: milk, composition, Maillard reaction, fluorescence spectroscopy

\section{Short Communication}

Milk is susceptible to changes that affect its organoleptic properties and nutritional quality because of chemical and biochemical reactions that occur following thermal treatment and storage. The Maillard reaction

Received April 21, 2011.

Accepted July 31, 2011.

${ }^{1}$ Corresponding authors: zhoupeng@jiangnan.edu.cn and liuxm@ jiangnan.edu.cn
(MR), generally described as a series of nonenzymatic reactions occurring in foods rich in reducing sugars and proteins, is one of the key chemical reactions in milk affecting its quality. Different compounds formed at various stages of this process, such as furosine and 5-hydroxymethylfurfural, have been used to track the extent of MR in foods (Kulmyrzaev and Dufour, 2002). However, most methods used to measure MR compounds are labor-intensive and time-consuming, and thus new methods must be developed that can rapidly detect the accumulation of MR compounds during processing and storage of foods. Front-face fluorescence spectroscopy (FFFS) has recently been used to monitor biochemical reactions in various food products due to its sensitivity, speed, and ability to assess the changes noninvasively and nondestructively (Kulmyrzaev et al., 2005).

Food quality is also associated with geographical origin. Today, consumers are paying more attention to the country of origin of foods when they purchase food items. However, the complexity of the food matrix and variability in raw materials has made the determination of product authenticity very challenging. Hence, choosing an appropriate and sensitive indicator and detection technique is critical. In China, UHT milks of various compositions account for the greatest portion in the category of liquid dairy products. Therefore, the purpose of this study was to develop a rapid technique using FFFS to evaluate the influence of composition and processing conditions on domestic and international commercial liquid milk, and to discriminate samples with different compositions and countries of origin. Chemical compounds such as MR products, riboflavin, and tryptophan were selected as indicators. The glycation extent of $\alpha$-LA in the samples was determined by liquid chromatography-mass spectrometry (LC-MS), and the relationship between the fluorescence spectral data and LC-MS data was investigated.

Six domestic and 7 international brands of pasteurized and UHT milk of various compositions were obtained from local supermarkets. The milk samples were designated with single letters according to their countries 
of origin: France $(\mathbf{F})$, New Zealand $(\mathbf{Z})$, Australia (A), China (C), and Germany (G). Sixty-six UHT (U) milk samples of varying compositions: whole $(\mathbf{W})$, skimmed $(\mathbf{S})$, reduced fat $(\mathbf{R})$, low lactose reduced fat $(\mathbf{L})$, high protein $(\mathbf{H})$, and 10 pasteurized $(\mathbf{P})$ milk samples were obtained. Fluorescence spectra were recorded using a F-7000 FL spectrophotometer (Hitachi High Technology, Tokyo, Japan) with the incidence angle of excitation of $56^{\circ}$ and the excitation and emission slits set at $5 \mathrm{~nm}$. The emission spectra of Maillard reaction products (MRP) and riboflavin (RF) (400 to $600 \mathrm{~nm}$ ), and tryptophan (300 to $400 \mathrm{~nm}$ ) were recorded using FFFS in duplicates at room temperature. The excitation wavelengths were set at $360 \mathrm{~nm}$ for $\mathrm{MRP} / \mathrm{RF}$ and $290 \mathrm{~nm}$ for tryptophan. A single scan was performed for $\mathrm{MRP}$ and RF (MRP/RF) because of their close excitation wavelengths (360 and $380 \mathrm{~nm}$, respectively), as reported by Liu and Metzger (2007). The tryptophan emission spectra were recorded according to Dufour and Riaublanc (1997) with slight modifications. All spectral scans recorded for each sample were averaged and normalized to reduce the scattering effect. The shift of the peak maximum values and the changes of peak width in the spectra were considered after normalization. Principal component analysis (PCA) was applied separately to the normalized spectral regions corresponding to $\mathrm{MRP} / \mathrm{RF}$ and tryptophan to obtain the score maps and spectral patterns. The statistical analysis was carried out using Unscrambler 9.7 (CAMO Software, Domlur, India).

The LC-MS measurement was conducted on whey protein fractions of 26 milk samples. The milk serum was removed after centrifugation at $10,000 \times g$ at $4^{\circ} \mathrm{C}$ for $20 \mathrm{~min}$. The whey proteins were then separated from caseins by acidic precipitation via addition of 2 $N \mathrm{HCl}$ to a final $\mathrm{pH}$ of 4.6 followed by centrifugation. The samples were analyzed for the degree of glycation by LC-MS using a Waters Acquity UPLC coupled with a time-of-flight mass spectrometer and an electrospray ion source. All the LC-MS components used were developed by Waters Corp. (Milford, MA) unless otherwise stated. The LC separation was performed on a $\mathrm{BEH}$ C18 column $(2.1 \times 50 \mathrm{~mm})$ at a column temperature of $45^{\circ} \mathrm{C}$. The solvents were $100 \%$ acetonitrile (A) and water containing $0.1 \%$ formic acid (B). The flow rate was $300 \mu \mathrm{L} / \mathrm{min}$ with a linear gradient from 80 to $50 \% \mathrm{~B}$ in $8 \mathrm{~min}$ and 50 to $0 \% \mathrm{~B}$ in the next $1 \mathrm{~min}$. The amount injected for each sample was $30 \mu \mathrm{L}$. The MS analysis was done in a positive mode scanning from $\mathrm{m} / \mathrm{z} 600$ to 3,000, and MassLynx V4.1 was used to analyze the spectra.

The normalized emission spectra of $\mathrm{MRP} / \mathrm{RF}$ displayed 2 spectral regions (around $420 \mathrm{~nm}$ and $516 \mathrm{~nm}$ ) with only minor variations in emission maximum wave- lengths and fluorescence intensity (data not shown). The spectral changes at $516 \mathrm{~nm}$ could be ascribed to the photodegradation of $\mathrm{RF}$, leading to formation of a fluorescent product, as reported by Wold et al. (2002). The peak at $420 \mathrm{~nm}$ was attributed to MRP, as indicated by Kulmyrzaev and Dufour (2002). Pasteurized milk displayed the highest intensity at $516 \mathrm{~nm}$ but the lowest intensity at $420 \mathrm{~nm}$ among all samples, which could be related to the different extents of heat treatment applied to the $\mathrm{P}$ and $\mathrm{U}$ milks.

The first 2 principal components (PC) from the PCA for MRP/RF were used to characterize the samples; the similarity map and factor loadings are shown in Figure 1. According to PC1 and PC2 (which accounted for 93 and $6 \%$ of the total data variability, respectively), the $\mathrm{P}$ milks clustered at the left side of the similarity map with negative scores, whereas the UHT skimmed (S) samples mainly had positive scores (Figure 1a). In the similarity map, the European samples ( $F$ and $G$ ) were discriminated from the brands from Australia and New Zealand ( $\mathrm{A}$ and $\mathrm{Z}$ ), with the $\mathrm{A}$ and $\mathrm{Z}$ samples having higher $\mathrm{PC} 2$ scores than the European brands. The domestic brands (C) grouped together near the center of the plot.

The factor loadings corresponding to $\mathrm{PC} 1$ presented opposition between a positive band at $430 \mathrm{~nm}$ and a negative band at $520 \mathrm{~nm}$ (Figure 1b). This could be attributed to the presence and variation of MRP in the samples, in agreement with the report by Liu and Metzger (2007) that ascribed such display to the accumulation of advanced glycation end-products during storage of dairy products. The factor loadings for PC2 displayed opposition between a negative peak at around $470 \mathrm{~nm}$ and a broad positive band at around $522 \mathrm{~nm}$, which was attributed to the photodegradation of riboflavin. Karoui et al. (2007) indicated that photodegradation of riboflavin leads to formation of lumichrome and lumiflavin, which emit between 444 and $479 \mathrm{~nm}$ and between 516 and $522 \mathrm{~nm}$, respectively. Therefore, PC2 expressed changes in the RF peak at $516 \mathrm{~nm}$ on the normalized spectra. A smaller peak was also observed at $406 \mathrm{~nm}$, but no literature was found that accounted with certainty for the fluorophores responsible. However, Karoui et al. (2007) and Wold et al. (2006) associated the peak of this nature to fluorescence from stable oxidation products, resulting from aldehydes and amino acids. Therefore, the results indicate that the MRP/RF spectrum discriminated the fluid milk according to the degree of Maillard reaction and photodegradation of RF due to processing conditions and composition.

The tryptophan emission spectra displayed a maximum peak at around $336 \mathrm{~nm}$ (data not shown). The maximum emission of tryptophan has been reported 

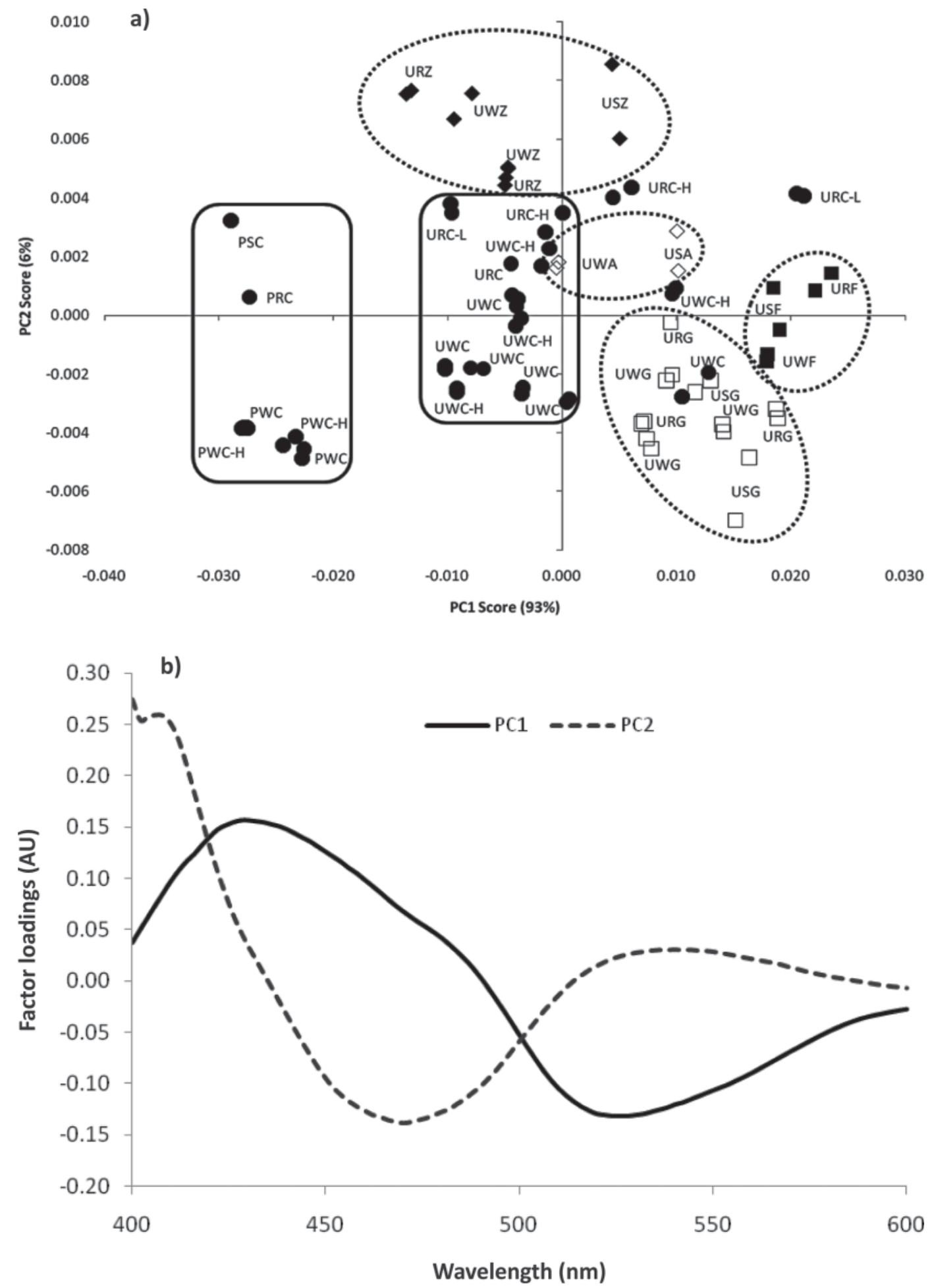

Figure 1. Principal component (PC) analysis similarity map (a) and factor loadings (b; arbitrary units) of Maillard reaction products and riboflavin spectra. The first letter in the sample name indicates the type of milk $(\mathrm{P}=$ pasteurized; $\mathrm{U}=\mathrm{UHT})$, the next letter represents milk composition ( $\mathrm{W}=$ whole; $\mathrm{S}=$ skim; $\mathrm{R}=$ reduced fat), and the last letter (and symbol) refers to the country of origin $[\mathrm{Z}=\mathrm{New} Z \mathrm{Zealand}$ ( $)$ ); $=$ Australia $(\diamond) ; \mathrm{F}=$ France $(\boldsymbol{\square}) ; \mathrm{G}=$ Germany $(\square) ; \mathrm{C}=$ China $(\bullet)]$. The hyphenated letter indicates milk samples with special composition $(\mathrm{L}=$ low lactose; $\mathrm{H}=$ high protein). 

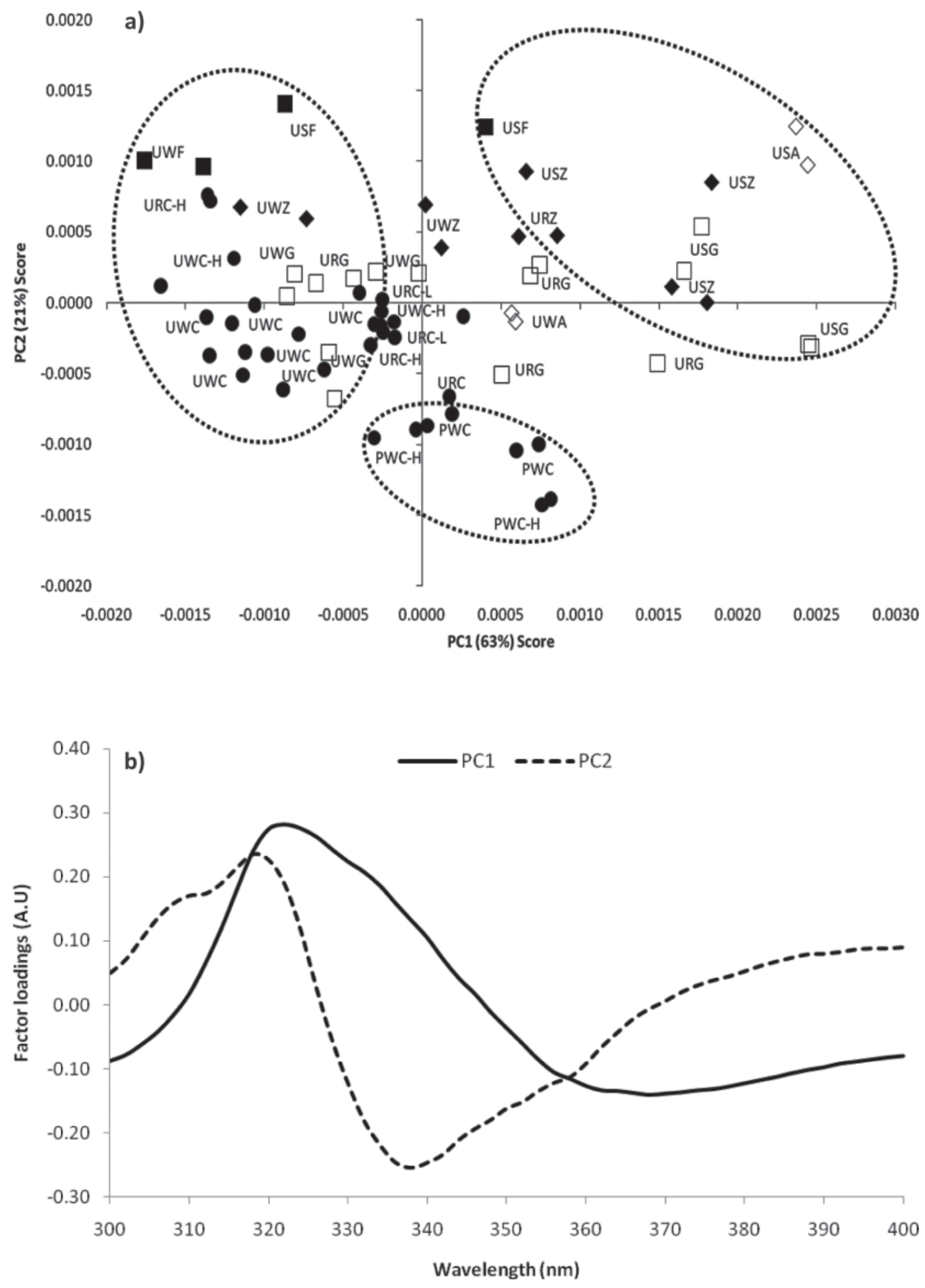

Figure 2. Principal component (PC) analysis similarity map (a) and factor loadings (b; arbitrary units) of tryptophan spectra. The first letter in the sample name indicates the type of milk $(\mathrm{P}=$ pasteurized; $\mathrm{U}=\mathrm{UHT})$, the next letter represents milk composition $(\mathrm{W}=$ whole; $\mathrm{S}$ $=$ skim; or $\mathrm{R}=$ reduced fat), and the last letter (and symbol) refers to the country of origin $[\mathrm{Z}=$ New Zealand $(\bullet)$; $\mathrm{A}=\mathrm{Australia}(\diamond) ; \mathrm{F}=$ France $(\square)$; $\mathrm{G}=$ Germany $(\square)$; C = China $(\bullet)$ ]. The hyphenated letter is used for milk samples with special composition $(\mathrm{L}=$ low lactose; H $=$ high protein). 

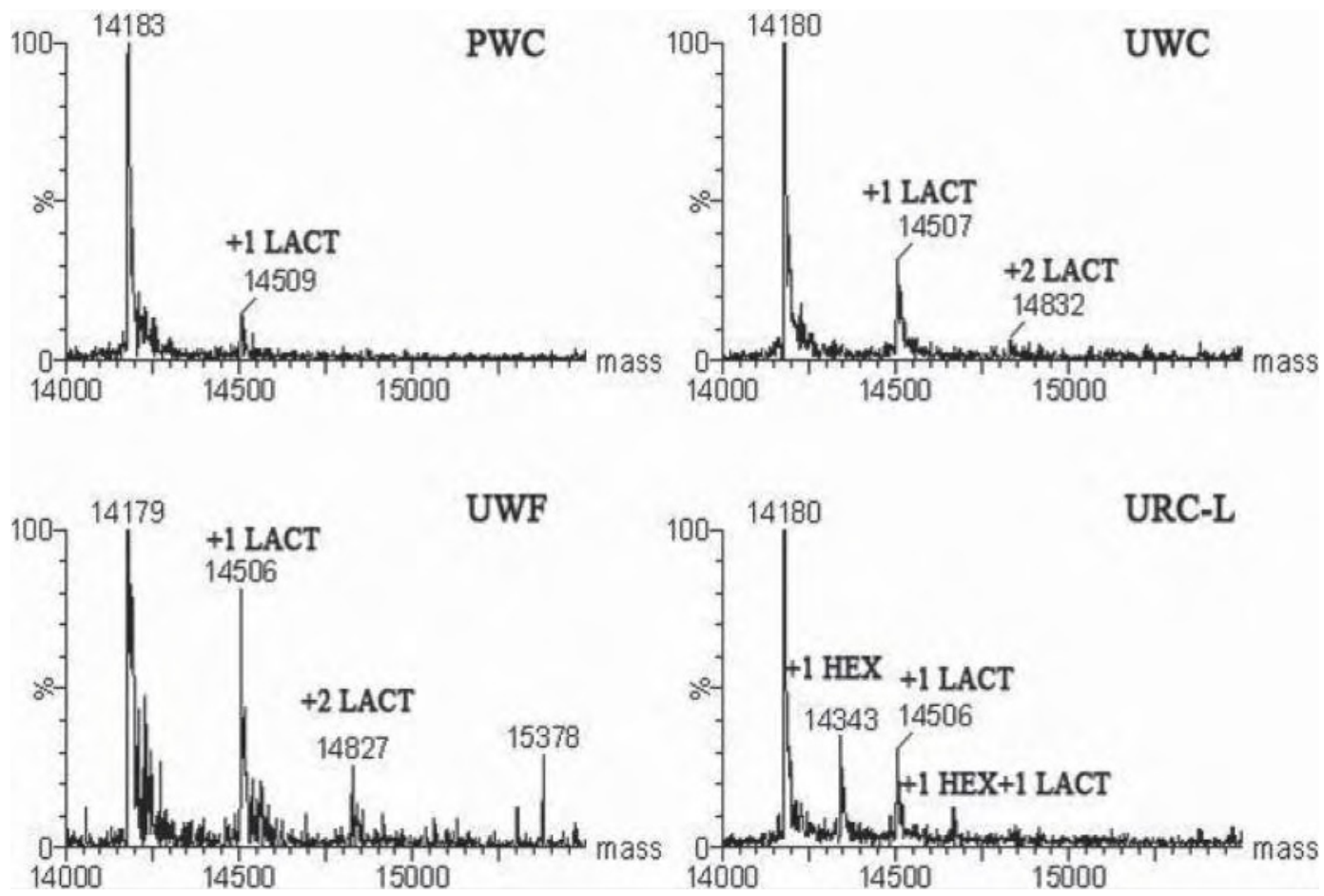

Figure 3. Mass spectra of $\alpha$-LA isolated from selected milk products. The first letter in the sample name indicates the type of milk $(\mathrm{P}=$ pasteurized; $\mathrm{U}=\mathrm{UHT})$, the next letter represents milk composition $(\mathrm{W}=$ whole; $\mathrm{R}=$ reduced fat), and the last letter refers to the country of origin ( $\mathrm{F}=$ France; $\mathrm{C}=$ China). The hyphenated letter is used for milk samples with special composition $(\mathrm{L}=$ low lactose). Peak identifications: +1 and +2 LACT indicate addition of 1 or 2 lactose molecules, respectively, to a protein amino group through Maillard reaction; +1 HEX indicates addition of 1 hexose molecule.

in most studies to be in the region between 332 and $343 \mathrm{~nm}$ (Dufour et al., 2001; Kulmyrzaev et al., 2005), depending on the samples and the processing and storage conditions. The PCA similarity map for tryptophan discriminated the samples according to PC1 (which accounted for $63 \%$ of the total data variability), PC2, and PC3 (which accounted for 21 and $12 \%$ of the total data variability, respectively). The PCA results indicated that PC1 mainly correlated with the composition (fat content) of the samples, with U skimmed (S) milk samples clustered together on the right side of the figure, with the UW milk and UR samples on the left side of the UHT skimmed (US) milk samples (Figure 2a). According to PC2, pasteurized milks had the lowest negative scores, and the majority of the UHT milk could be separated as international samples (positive PC2 scores) and domestic samples (negative PC2 scores).

The factor loadings for PC1 and PC2 corresponding to tryptophan are shown in Figure 2b. The factor loadings for $\mathrm{PC} 1$ presented opposition between a positive peak at around $320 \mathrm{~nm}$ and a broad negative band at around $362 \mathrm{~nm}$. The factor loadings for PC2 displayed a shift to shorter wavelengths with an opposition between a positive peak at $318 \mathrm{~nm}$ and a negative peak at around $338 \mathrm{~nm}$. In a previous study by Dufour and Riaublanc (1997), a blue shift of the maximum emission of proteins induced by homogenization was reported. The fluorescence properties of tryptophan are sensitive to the microenvironment surrounding the tryptophan residues, and the variation in composition and processing conditions in the samples could result in changes in fluorescence intensity and shifts in the emission maxima to shorter wavelengths.

Analysis by LC-MS was used to assess the extent of protein glycation in selected samples of different compositions. $\alpha$-Lactalbumin was clearly detected in the samples, and the exemplary deconvoluted mass spectra of 4 different samples are shown in Figure 3. The first peak in the spectrum displayed the calculated molecular mass of 14,179 to $14,183 \mathrm{Da}$, which is very close to the expected value for $\alpha$-LA $(14,178.0 \mathrm{Da})$, as indicated by Marvin et al. (2002), and was therefore attributed to $\alpha$-LA. The degree of glycation was determined according to Czerwenka et al. (2006). The determination of an overall extent of glycation reaction from the intensities of various glycated and nonglycated species in the deconvoluted mass spectra was derived by calculating the degree of glycation as the average number of lactose molecules bound to 1 protein molecule. In the previ- 


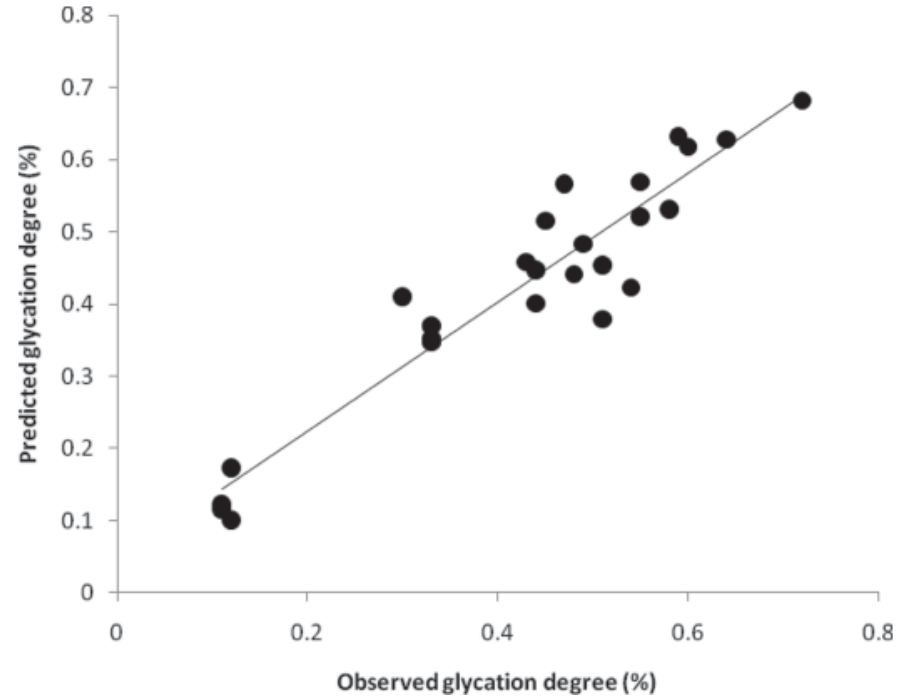

Figure 4. Predicted and observed glycation degree of pasteurized and UHT milk samples with different compositions based on a principal component regression model taking into account of 3 principal components of Maillard reaction products and riboflavin spectra and liquid chromatography-mass spectrometry data.

ous study by Marvin et al. (2002), $\alpha$-LA was preferred over $\beta-\mathrm{LG}$ as the biomarker, and the mass difference of $324 \mathrm{Da}$ was due to the addition of a lactose moiety on a protein amino group through the Maillard reaction. The pasteurized milks in this study displayed the lowest extent of glycation due to low processing temperatures, as reported by Czerwenka et al. (2006). The various UHT whole milks showed variations in intensities and mass increases, probably due to different industrial processing conditions.

Finally, principal component regression analysis was applied to the data matrices of PCA results of FFFS (MRP/RF and tryptophan) and the LC-MS data. A strong correlation between the LC-MS data and the $\mathrm{MRP} / \mathrm{RF}$ data (specifically $\mathrm{PC} 1$ ) was found, which showed that PC1 discriminated the samples according to the extent of glycation. The observed and predicted values for the degree of glycation were clearly described by a linear regression $\left(R^{2}=0.84\right)$, as shown in Figure 4 .
Front-face fluorescence spectroscopy is able to rapidly characterize commercial fluid milk samples for extent of glycation and microenvironment of tryptophan in dairy proteins. Additional studies with a larger sample size would be useful to identify the specific factors responsible for the variation in fluid milks observed by FFFS.

\section{ACKNOWLEDGMENTS}

This work was supported by the National Natural Science Foundation of China (No. 30901128 and 31071492), the Science and Nature Foundation in Jiangsu Province (No. BK2009070), and the 111 project B07029.

\section{REFERENCES}

Czerwenka, C., I. Maier, F. Pittner, and W. Linder. 2006. Investigation of the lactosylation of whey proteins by liquid chromatography-mass spectroscopy. J. Agric. Food Chem. 54:8874-8882.

Dufour, E., M. F. Devaux, P. Fortier, and S. Herbert. 2001. Delineation of the structure of soft cheeses at the molecular level by fluorescence spectroscopy_Relationship with texture. Int. Dairy J. 11:465-473.

Dufour, E., and A. Riaublanc. 1997. Potential of spectroscopic methods for the characterization of dairy products. I. Front-face fluorescence study of raw, heated and homogenized milks. Lait 77:657670.

Karoui, R., E. Dufour, and J. D. Baerdemaeker. 2007. Front face fluorescence spectroscopy coupled with chemometric tools for monitoring the oxidation of semi-hard cheeses throughout ripening. Food Chem. 101:1305-1314.

Kulmyrzaev, A., and E. Dufour. 2002. Determination of lactulose and furosine in milk using front-face fluorescence spectroscopy. Lait $82: 725-735$

Kulmyrzaev, A., D. Levieux, and E. Dufour. 2005. Front-face fluorescence spectroscopy allows the characterization of mild heat treatment applied to milk. Relations with the denaturation of milk proteins. J. Agric. Food Chem. 53:502-507.

Liu, X., and L. E. Metzger. 2007. Application of fluorescence spectroscopy for monitoring changes in non-fat dry milk during storage. J. Dairy Sci. 90:24-37.

Marvin, L. F., V. Parisod, L. B. Fay, and P. A. Guy. 2002. Characterization of lactosylated proteins of infant formula powders using two dimensional gel electrophoresis and nanoelectrospray mass spectrometry. Electrophoresis 23:2505-2512.

Wold, J. P., K. Jorgensen, and F. Lundby. 2002. Nondestructive measurement of heat-induced oxidation of dairy products by fluorescence spectroscopy and imaging. J. Dairy Sci. 85:1693-1704.

Wold, J. P., A. Veberg, F. Lundboy, A. N. Nilsen, and J. Moan. 2006. Influence of storage time and color of light on photooxidation in cheese: A study based on sensory analysis and fluorescence spectroscopy. Int. Dairy J. 16:1218-1226. 